\title{
Switching processes and switching reproducibility in ferromagnetic ring structures
}

\author{
M. Kläui, ${ }^{\text {a) }}$ C. A. F. Vaz, and J. A. C. Bland ${ }^{\text {b) }}$ \\ Cavendish Laboratory, University of Cambridge, Madingley Road, Cambridge, CB3 OHE, United Kingdom \\ E. H. C. P. Sinnecker and A. P. Guimarães \\ Centro Brasileiro de Pesquisas Físicas, R. Xavier Sigaud 150, Rio de Janeiro, 22290-180, Brazil
}

W. Wernsdorfer

Laboratoire L. Néel-CNRS, BP 138, 38274 Grenoble, France

G. Faini and E. Cambril

Laboratoire de Photonique et de Nanostructures-CNRS, Route de Nozay, 91460 Marcoussis, France

L. J. Heyderman and C. David

Laboratory for Micro- and Nanotechnology, Paul Scherrer Institut, CH-5232 Villigen PSI, Switzerland

(Received 8 October 2003; accepted 17 November 2003)

\begin{abstract}
We present an experimental study of the reproducibility of the different switching processes occurring in rings. Using superconducting quantum interference device and magnetoresistance measurements, we can measure hysteresis loops of arrays of rings and single structures at varying temperatures and thereby separate the influence of thermal excitations and defects (extrinsic and intrinsic). We find that the temperature dependence of the switching fields and their distributions can be correlated with the different physical processes occurring during different transitions. Measurements of the angular dependence of the switching fields of a single ring allow us to distinguish the contributions of extrinsic and intrinsic defects to the switching field distributions and, counterintuitively, it is established that transitions involving nucleation processes are less prone to defects and thermal excitations than processes involving domain-wall or vortex core depinning and propagation. (C) 2004 American Institute of Physics. [DOI: 10.1063/1.1640451]
\end{abstract}

Small magnetic structures have been widely proposed for applications in nonvolatile memory devices and magnetosensors. ${ }^{1,2}$ The equilibrium magnetic states and magnetization reversal mechanisms in small elements, are strongly determined by the interplay of magnetic anisotropies (e.g., magnetocrystalline, interface, etc.) with the physical shape of the element. ${ }^{3-10}$ The critical property for applications is the magnetic switching. To ascertain how thermal excitations and extrinsic and intrinsic defects affect the switching processes, one requires a geometry where transitions by different physical processes occur and also a thorough understanding of these processes. A possible geometry that fulfills these criteria is the ring geometry. Rings exhibit a range of switching behaviors, from single onion to reverse onion, switching via a double switching process (onion to vortex and vortex to reverse onion) and a triple switching involving the vortex core state. ${ }^{3,6,7}$ These different transitions involve physically very different processes (some are nucleation free, some involve nucleation of domains or vortex cores, etc.). ${ }^{8}$ There are a number of different factors that contribute to switching field distributions. At room temperature, thermal excitations can overcome energy barriers and cause elements to switch, and in addition, intrinsic and extrinsic defects will produce different switching fields for different structures.

Arrays of polycrystalline Co rings were fabricated as

\footnotetext{
${ }^{a)}$ Current address: Fachbereich Physik, Universitaet Konstanz, Universitaetsstr. 10, D-78457 Konstanz, Germany.

${ }^{\text {b) }}$ Author to whom correspondence should be addressed; electronic address: jacb1@phy.cam.ac.uk
}

presented and shown in Ref. 7. As explained in Ref. 6, very wide and thick rings (outer diameter $D=1.6 \mu \mathrm{m}$, width $W=700 \mathrm{~nm}$ and thickness $t=34 \mathrm{~nm}$ ) exhibit the most complex switching (three transitions), as also presented in Fig. 1(a). Comparing the results of the micromagnetic simulation (open circles) with the superconducting quantum interference device (SQUID) measurement shows good agreement. The three transitions between the magnetic states shown in the schematic insets are: (i) Onion [Fig. 1(a)] to vortex, [Fig. 1(b)] transition, which is a domain-wall depinning and propagation process; ${ }^{3}$ (ii) the vortex [Fig. 1(b)] to vortexcore [Fig. 1(c)] transition, which involves a nucleation of a vortex core at the inner edge; ${ }^{8}$ (iii) the vortexcore [Fig. 1(c)] to reverse onion [Fig. 1(d)] transition, which is a vortex core annihilation process. ${ }^{8}$

As the temperature is decreased to $5 \mathrm{~K}$, the magnetic switching fields and switching fields distributions change as thermal excitations are reduced as presented in Fig. 1. Considering the first transition (onion to vortex), it can be seen that at $5 \mathrm{~K}$, the onion to vortex switching field $H_{c 1}$ is around $45 \mathrm{Oe}$, whereas at high temperatures the rings fall into the vortex state when the field is lowered before reaching $H=0$ Oe. The physical process (domain-wall depinning and propagation) gives a possible explanation in that the domain wall is pinned at local defects (intrinsic or extrinsic as discussed below), which represent energy barriers between two energy minima. These barriers need to be overcome for the wall to depin and the switching to occur. At high temperatures, the thermal excitations help to overcome these energy barriers thereby leading to earlier reversal. 

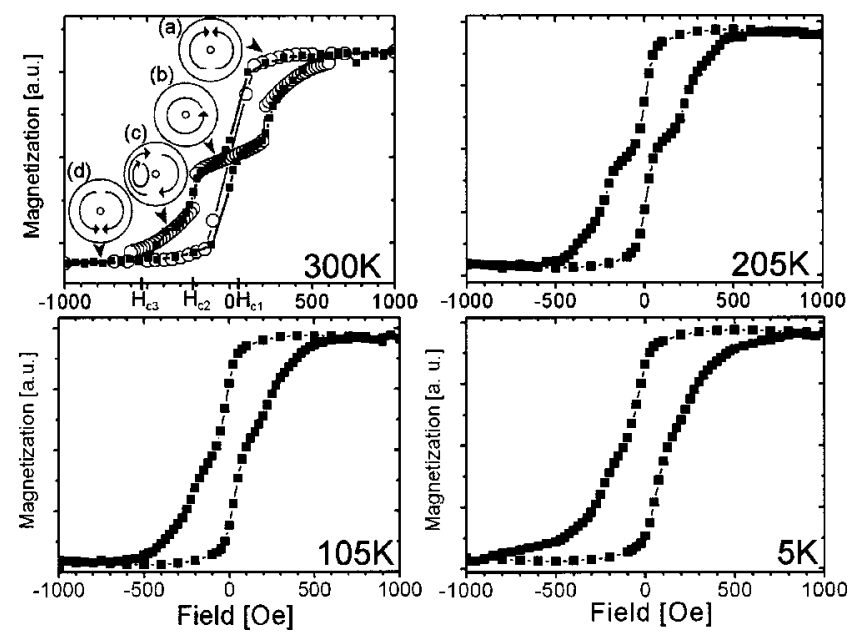

FIG. 1. Hysteresis loops of an array of polycrystalline cobalt rings (outer diameter $D_{\text {ext }}=1.7 \mu \mathrm{m}$, width $W=700 \mathrm{~nm}$, and thickness $t=34 \mathrm{~nm}$ ) taken at $300 \mathrm{~K}, 205 \mathrm{~K}, 105 \mathrm{~K}$, and $5 \mathrm{~K}$. In the $300 \mathrm{~K}$ measurement, the magnetization configurations are shown schematically [onion state (a), vortex state (b), vortexcore state (c) and reverse onion state (d)] and the results for micromagnetic simulations are added (empty disks). A large change in the switching fields between the low- and the high-temperature measurements can be observed for some of the transitions.

The transition for the vortex to the vortexcore state occurs at $T=300 \mathrm{~K}$ at around $H_{c 2}=210 \mathrm{Oe}$ (Fig. 1), whereas at $5 \mathrm{~K}$ it occurs at a slightly higher switching field of around 230 Oe. From this, it can be deduced that this switching process, which is a nucleation of a vortexcore at the inner edge of the ring, ${ }^{8}$ is only affected little by thermal excitations. In contrast to the depinning of a domain wall where a number of small barriers is present due to local pinning sites for the wall, this nucleation process does not depend on the pinning of a domain wall or vortex core.

Finally the maximum vortexcore to reverse onion transition field is $H_{c 3}=530 \mathrm{Oe}$ at $T=300 \mathrm{~K}$ and around $850 \mathrm{Oe}$ at $5 \mathrm{~K}$. This large increase shows that the annihilation of the vortex core (in contrast to its nucleation) is strongly assisted by thermal excitations. This can again be understood by the fact that the vortex core is pinned at defects due to its high exchange energy. Again, this pinning leads to small energy barriers, which can be overcome by thermal fluctuations, leading to an earlier annihilation (pushing out) of the vortexcore for high temperatures. At low temperatures, stronger fields are needed to overcome the pinning and annihilate the vortex core.

Considering the switching field distributions, it can be seen that all the switching field distributions increase with decreasing temperature. Similar to the change in switching field, it can be seen that the onion to vortex and the vortex core to reverse onion switching field distributions are strongly broadened when the temperature is decreased to $5 \mathrm{~K}$ (respectively, by $30 \%$, and $40 \%$ increase in the relative full width half maximum of the distribution divided by the average switching field), whereas the vortex to vortexcore switching field distribution only changes a little $(10 \%$ increase). In the case of processes involving the depinning of a domain wall (onion to vortex) and the annihilation of a vortex core (vortexcore to reverse onion) the pinning of the domain wall, and the vortex core is different (the local energy barriers are different) for each element due to differences in the defects. So at low temperatures, these two tran- sitions will have a large switching field distribution reflecting the differences in energy barrier heights. This switching field distribution is reduced at a high temperature due to the thermal excitations overcoming the small local energy barriers.

It is interesting to note that the micromagnetic calculations simulate a perfect ring without defects (apart from the discretization induced edge roughness) and do not include any thermal excitations $(0 \mathrm{~K})$, but reproduce the measurement at high temperatures much better than the lowtemperature measurement as seen in Fig. 1. This means that the defects that hinder switching at low temperatures in the experiment are being overcome by the thermal excitations. So the ring behaves much more like a "perfect" ring at elevated temperatures, while at low temperatures for some of the transitions, local defects, which are not included in the simulations, become effective and govern the reversal fields.

Having discussed the rather complex triple switching, we now turn to the most common switching process, which is the case of double switching (onion to vortex and vortex to reverse onion). ${ }^{3,5,10}$ The onion to vortex switching is a wall depinning and propagation process, ${ }^{3,4}$ whereas the vortex to reverse onion is a nucleation process, followed by the spread of a reverse domain. ${ }^{5}$ This difference has implications for the reproducibility and hence the switching field distribution. As seen in Refs. 3 and 6, the switching field distribution for the onion to vortex transition is larger than that for the vortex to reverse onion transition. This can be understood as discussed above, taking into account that the domain-wall depinning field (i.e., the switching field for the onion to vortex transition) is highly dependent on the pinning strength of the defect it is pinned at (which will vary from ring to ring and thus lead to a large switching field distribution). Temperature dependent measurements on an array of polycrystalline Co rings exhibiting a double switching (outer diameter $D=1.6$ $\mu \mathrm{m}$, width $W=250 \mathrm{~nm}$, and thickness $t=34 \mathrm{~nm}$ ) show, similarly to the results for triple switching discussed above, that the onion to vortex transition is strongly assisted by thermal excitations (lower switching field and smaller switching field distribution at high temperatures). The vortex to reverse onion transition (nucleation and spread of a reverse domain process) is less affected by temperature variations. We turn now to the issue of how to separate the contributions from extrinsic and intrinsic defects, achieved by studying the behavior of single rings at low temperatures, where thermal excitations are suppressed. Since rings have an in-plane rotational symmetry, measuring the angular dependence of the switching fields yields information about the switching field distribution within a ring due to shape irregularities and intrinsic defects. Permalloy rings with nonmagnetic contacts and no artificial asymmetries were fabricated as detailed in Ref. 4 [a scanning electron microscope (SEM) image is shown in Fig. 2] and four-probe magnetoresistance measurements were carried out at $4 \mathrm{~K}^{4}$

The resistance is measured between opposite contacts to obtain the two switching fields (onion to vortex and vortex to onion) for all angles $0^{\circ}-180^{\circ}$ (as positive and negative fields are measured, this effectively means that values for $0^{\circ}-360^{\circ}$ are obtained). In Fig. 3, the onion to vortex and vortex to reverse onion switching fields are plotted as a function of angle. As also observed in the MOKE and SQUID measure- 


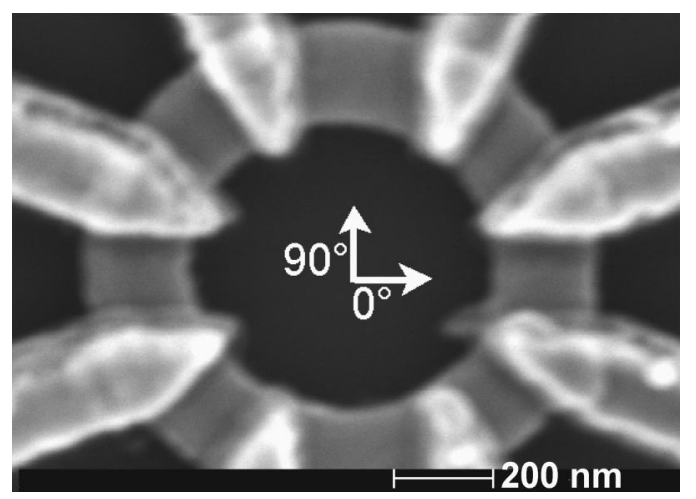

FIG. 2. SEM image of a ring $\left(D_{\mathrm{ext}}=1 \mu \mathrm{m}, W=200 \mathrm{~nm}, t=34 \mathrm{~nm}\right.$ Py) with no artificial defects and eight nonmagnetic contacts.

ments of arrays at room temperature, the onion to vortex switching field distribution is much larger than the vortex to reverse onion switching field distribution, which can be explained by the fact that the domain-wall depinning of the onion to vortex switching process is very defect dependent. What is striking in this graph, is the fact that for the vortex to reverse onion transition, the positive and negative switching fields are virtually always the same, whereas the onion to vortex switching fields vary greatly. This means that the very similar variation of the positive and negative vortex to onion switching fields with angle are primarily due to shape variations (which affect the switching fields for positive and negative switching fields equally). This can be explained from the switching mechanism, since the nucleation of a reverse domain happens at the edge of the ring ${ }^{10}$ and is thus very shape dependent.

For the onion to vortex switching though, the variation between positive and negative switching fields is of the same order as the variation between different angles. It should be noted that the switching fields are quite reproducible (the error bars are of the order of a few tens of Oe) so that the difference between positive and negative switching fields is reproducible and cannot be attributed to random processes. This means that neither extrinsic shape irregularities, nor random thermal excitation processes are the dominant factors here, but symmetry breaking intrinsic defects. Possible intrinsic defects are local oxidation spots, which can induce exchange bias effects: $\mathrm{Ni}$ and $\mathrm{Co}$ oxides become antiferromagnetic at low temperatures and can thus break the time symmetry and cause an exchange bias effect. ${ }^{11}$ Hence, at low temperatures for the onion to vortex transition, the pinning due to intrinsic defects is significant. Again this is perfectly reasonable taking into account that the switching field is dependent on the pinning of the domain wall and that exchange bias materials can exercise strong pinning on the magnetization in domain walls. From this, we can conclude that the spread of the switching field distribution for the onion to vortex transition is due to a combination of extrinsic shape variations and pinning due to exchange bias whereas the much smaller vortex to reverse onion transition switching field distribution is primarily due to shape variations. For applications, this means that improving the shape reproducibility will improve the vortex to onion transition, but this will benefit little the onion to vortex switching, where materials with less intrinsic defects are needed for a smaller switching field distribution.

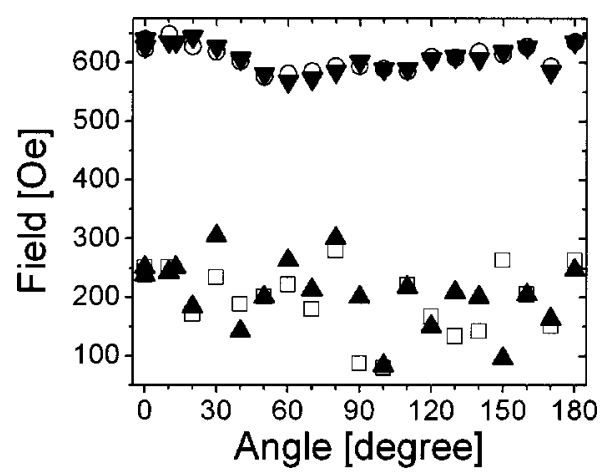

FIG. 3. Switching fields at $4 \mathrm{~K}$ for the onion to vortex transition (positive fields: Empty squares, negative fields: Up triangles) and the vortex to reverse onion transition (positive fields: empty disks, negative fields: Down triangles) of the ring shown in Fig. 2 as a function of the applied field angle. Note the different switching field spread for the two transitions.

In conclusion, we have investigated the influence of defects on the switching fields and switching field distributions in ferromagnetic ring structures as a function of temperature. Surprisingly, transitions involving nucleation of either a vortex core or a reverse domain show small switching field distributions compared with nucleation-free transitions occurring by domain-wall motion or vortex core annihilation. For the latter transitions, contributions of intrinsic defects and extrinsic shape variations are identified as being significant, whereas for transitions involving the nucleation of a reverse domain extrinsic shape variations are responsible for the switching field distribution. From this, we can infer that, in contrast to the intuitive idea that nucleation-induced reversals entail a large switching field distribution, the switching field distribution and susceptibility to thermal effects in fact compare favorably to that for pinning and propagation processes.

The authors acknowledge technical support by L. Couraud and X. Lafosse, support by the "Region Ile de France," the "Conseil General de l'Essonne," the European ESPRIT network MASSDOTS (No. 22464), the CMI Magnetoelectronic Devices Project, the Profix program from CNPq (EHCPS), and the Gottlieb Daimler- and Karl Benz-Foundation for one of the authors (M.K.).

${ }^{1}$ G. A. Prinz, J. Magn. Magn. Mater. 200, 57 (1999).

${ }^{2}$ M. M. Miller, G. A. Prinz, S.-F. Cheng, and S. Bounnak, Appl. Phys. Lett. 81, 2211 (2002).

${ }^{3}$ J. Rothman, M. Kläui, L. Lopez-Diaz, C. A. F. Vaz, A. Bleloch, J. A. C. Bland, Z. Cui, and R. Speaks, Phys. Rev. Lett. 86, 1098 (2001).

${ }^{4}$ M. Kläui, C. A. F. Vaz, J. A. C. Bland, W. Wernsdorfer, G. Faini, and E. Cambril, Appl. Phys. Lett. 81, 108 (2002).

${ }^{5}$ M. Kläui, C. A. F. Vaz, J. Rothman, J. A. C. Bland, W. Wernsdorfer, G. Faini, and E. Cambril, Phys. Rev. Lett. 90, 97202 (2003).

${ }^{6}$ M. Kläui, C. A. F. Vaz, L. Lopez-Diaz, and J. A. C. Bland, J. Phys.: Condens. Matter 15, 985 (2003).

${ }^{7}$ Y. G. Yoo, M. Kläui, C. A. F. Vaz, J. A. C. Bland, and L. J. Heyderman, Appl. Phys. Lett. 82, 2470 (2003).

${ }^{8}$ M. Kläui, C. A. F. Vaz, J. A. C. Bland, T. L. Monchesky, J. Unguris, E. Bauer, S. Cherif, S. Heun, A. Locatelli, L. J. Heyderman, and Z. Cui, Phys. Rev. B 68, 134426 (2003).

${ }^{9}$ C. A. F. Vaz, L. Lopez-Diaz, M. Kläui, T. L. Monchesky, J. Unguris, Z. Cui, and J. A. C. Bland, Phys. Rev. B 67, 140405 (2003).

${ }^{10}$ M. Kläui, L. Lopez-Diaz, J. Rothman, C. A. F. Vaz, J. A. C. Bland, and Z. Cui, J. Magn. Magn. Mater. 240, 7 (2002).

${ }^{11}$ P. Miltényi, M. Gierlings, J. Keller, B. Beschoten, G. Güntherodt, U. Nowak, and K. D. Usadel, Phys. Rev. Lett. 84, 4224 (2000). 